\title{
NUCLEOLAR CONTROLLED SEGREGATION OF THE SEX CHROMOSOMES IN BEETLES
}

\author{
B. JOHN \\ Genetics Department, Birmingham University \\ and \\ K. R. LEWIS \\ Botany Department, Oxford University
}

\section{INTRODUCTION}

Received 24.ix.60

THE commonest method of diploid sex determination depends on the segregation of distinctive chromosomes which are homozygous in one sex and heterozygous in the other. Specific pairing followed by chiasma formation is both a sufficient and common means of achieving segregation in such systems. But chiasmata are not indispensable and they may be supplemented or replaced by other devices which allow the sex chromosomes to undergo regular segregation in the heterogametic sex. It is interesting to note, however, that in very few of the cases where chiasmata are lacking is bivalent formation also dispensed with.

Chiasmate and non-chiasmate associations between sex chromosomes are not always easy to distinguish. This applies to the unique parachute-type sex bivalent found in the males of at least twenty families of the Coleoptera (Smith, I953). These bivalents consist of a relatively large metacentric $\mathrm{X}$ chromosome associated with an invariably smaller and often minute $y_{p}$ chromosome. Smith (I95I) has tentatively advanced the view that these unequal chromosomes are associated at metaphase by two terminal chiasmata. One obvious factor which suggests that this may not be the case is the very small size of the $y$ chromosome.

In 1957 we reported a multiple sex-chromosome system in the cellar beetle, Blaps mucronata (Coleoptera: Tenebrionida), where segregation depended, in part, on an association between the sex chromosomes and a persistent nucleolus (Lewis and John, I957). This case has led us to consider whether a similar mechanism operates also in $\mathrm{X} y_{p}$-coleopterans which belong to the presumed ancestral type.

To test this we have examined males of the two-spotted ladybird (Adalia bipunctata) and the seven-spotted ladybird (Coccinella septempunctata), in which the $\mathrm{X} y_{p}$ metaphase bivalent is among the largest and the clearest we have seen. Males of Tenebrio molitor have also been used because, while the metaphase bivalent is less amenable to analysis, prophase stages are much clearer than in the ladybirds. All three species have the same diploid count consisting of $9 \mathrm{AA}+\mathrm{X} y_{p}$. 
Testes of these three species were freed from the investing fat body, fixed in $1: 3$ acetic-alcohol and stained in iron aceto-carmine, acetic orcein or by the Feulgen method. Aceto-carmine stains the nucleolus but the other methods do not.

\section{OBSERVATIONS}

The sex chromosomes are positively heteropycnotic and associated both in the pre-meiotic interphase nucleus and at early prophase. Their individuality cannot be recognised at this stage (plate, fig. I). The autosomes are also characterised by heterochromatic segments in the vicinity of their centromeres but unlike the sex chromosomes these

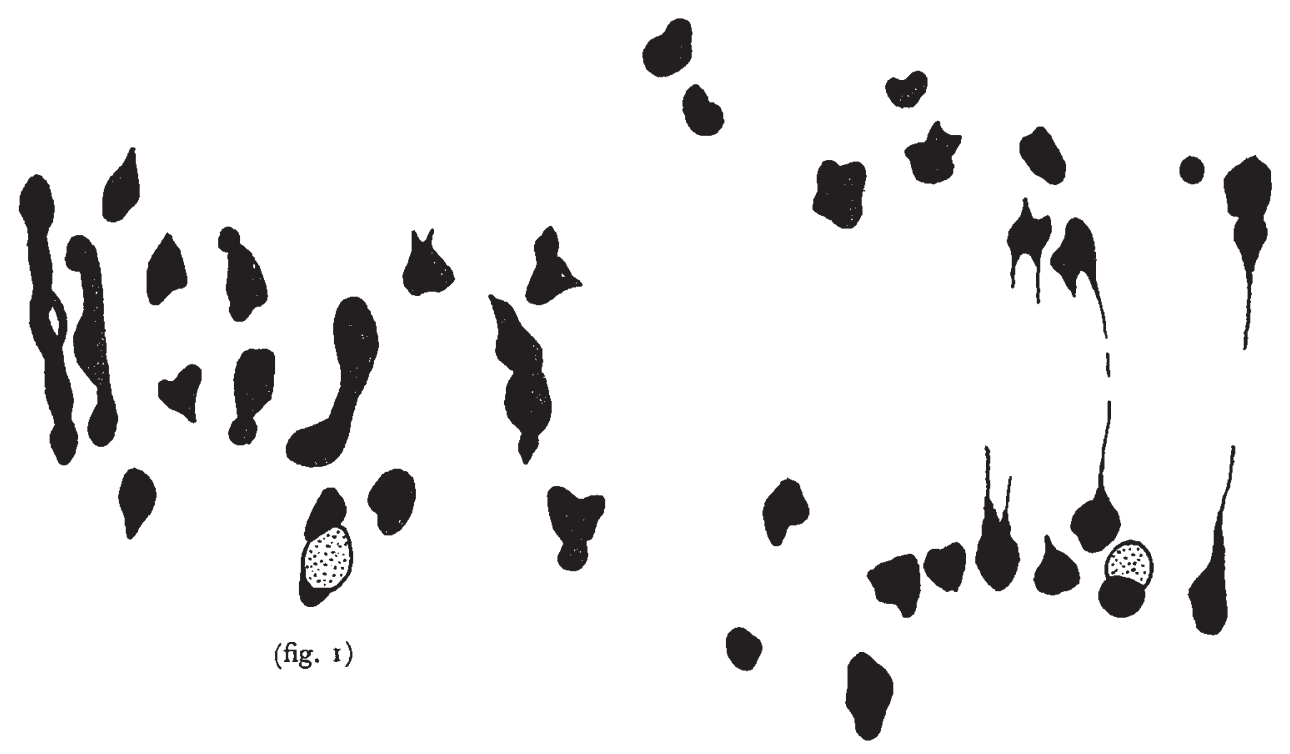

(fig. 2)

Text-Frgs. I and 2.-Adalia bipunctata. First metaphase (fig. I) and first anaphase (fig. 2) of meiosis. Note persistent nucleolus. $\times 4500$ (Aceto-carmine).

are not regularly associated before the completion of zygotene. Indeed these centric $\mathrm{H}$-segments, like those in Blaberus discoidalis (John and Lewis, 1959), are the last to pair. (plate, fig. I). At pachytene the sex-bivalent stains less deeply than at earlier stages. It is still in advance of the distal euchromatic regions of the autosomes but is less condensed than their centric heterochromatin (plate, fig. 2). At this time the sex bivalent frequently assumes a signet-ring appearance and is associated with nucleolar material which we have failed to demonstrate at earlier stages (plate, figs. 3 and 4 ). It would appear therefore that there is a correlation between the change in pycnosity and the organisation of a nucleolus.

By metaphase the sex chromosomes and the autosomes are similar in their staining reaction. Preparations stained in acetic orcein or 
feulgen show the typical parachute bivalent at this stage (plate, fig. 5). But with aceto-carmine the sex chromosomes are seen to be held together by a nucleolus (text-fig. I and plate, figs. 6-го). Usually this is found between the sex chromosomes; occasionally it is to one side, but this may be the effect of squashing.

At anaphase of the first meiotic division the $y_{p}$ chromosome becomes detached from the nucleolus and this organelle moves to the same pole as the $\mathrm{X}$ chromosome (text-fig. 2 and plate, fig. II). Like the persistent nucleolus of Blaps it does not disappear until first telophase.

We conclude that the formation of the $\mathrm{X}_{p}$-bivalent is due initially to the characteristic stickiness of heterochromatin. But the maintenance and regular segregation of this bivalent is due to the formation of a nucleolus during prophase and its persistence until segregation is ensured.

It is now possible to understand why congression of the sex bivalent is not infrequently delayed (plate, fig. 7 and cf. Smith, I952a and $b$ ) for the presence of a nucleolus must surely result in some degree of impedance to movement on the spindle.

\section{DISCUSSION}

\section{(i) Heterochromatic and nucleolar type sex-chromosome associations}

Reduction normally depends on bivalent formation which takes place in two stages:

(a) Specific pairing which leads to bivalent formation at zygotene.

(b) Chiasma formation which is responsible for maintaining the bivalent from diplotene to metaphase.

The second stage is conditional on the first but they are to some extent independently variable and the mechanism responsible for association at one stage is different from that at the other.

The inauguration of a sex-chromosome system on the other hand depends on a limitation of crossing-over and one of the commonest trends during the evolution of such a system is an increasing differentiation of the $\mathrm{X}$ and $\mathrm{Y}$ components. The differential segments extend at the expense of the pairing segments which may eventually be lost. In this event specific pairing and chiasma formation are both excluded.

Where the pairing segments are short the problem of ensuring zygotene association is increased. This is especially true when the differential segments are extensive for these, showing no tendency to specific pairing, are a "drag" to chromosome movement in the prophase nucleus. On a priori grounds therefore one might expect some means of facilitating specific pairing in such cases and a mechanism whose original mechanical function was merely to facilitate pairing could become modified during the course of evolution to maintain the metaphase association of completely differential sex chromosomes. What is subsidiary, incidental or even disadvantageous at one stage of evolution may prove indispensable at another. 
Various investigators have often been, indeed still are, in disagreement concerning the relationship of particular sex chromosomes and especially with regard to the possibility of a chiasmate association between them. But for the moment we are concerned only with their initial association.

In this connection a particularly instructive series of relationships between the $\mathrm{X}$ and $\mathrm{Y}$ chromosomes exists in rodents where an RNAcontaining sex vesicle is involved in their association. In Microtus agrestis, where the sex chromosomes are enormous compared with the autosomes, Sachs (I953) has concluded that the giant $\mathrm{X}$ and $\mathrm{Y}$ chromosomes are largely differential and that a chiasmate association forms in a small terminal pairing segment. Thus it appears that Microtus is an extreme example of the general case already considered. Now at leptotene the $\mathrm{X}$ and $\mathrm{Y}$ are both heteropycnotic but separate and each now organises its own nucleolar-like sex vesicle._- "The X and $\mathrm{Y}$ then move together until they are included in the one large vesicle at pachytene". Thus, initial association is by the fusion of nucleoli. Leptotene proximity is favoured by the lagging of the $\mathrm{X}$ and $\mathrm{Y}$ at anaphase of the spermatogonial mitoses and the somatic pairing occasionally observed in the spermatogonia may have the same utility.

In the mouse too it appears that the positively heteropycnotic sex chromosomes exist as separate entities at leptotene, are embedded in a common sex vesicle at zygotene and are associated end-to-end at pachytene. Here, however, it is not clear whether the sex chromosomes associate before or after the vesicle forms (cf. Ohno, Kaplan and Kinosita, I $959 b$ ). In the rat on the other hand, the sex chromosomes appear to be associated with a common sex vesicle "from the beginning" (Ohno, Kaplan and Kinosita, I956, I958). Where heterochromasy and nucleolar formation go together their individual roles are not easily distinguished, but this is possible to some extent in the earwig Forficula auricularia where the sex chromosomes are completely differential.

In Forficula the sex chromosomes "come together at telophase of the last spermatogonial mitosis. They are fully condensed when they associate and it is highly improbable that their association leads to chiasma formation" (Callan, I 94I). During the prophase of meiosis the sex chromosomes have a "covering of nucleolar material", but in $2 n=25$ chromosome-males, which are $\mathrm{X}_{1} \mathrm{X}_{2} \mathrm{Y}$, a bivalent and univalent condition of the sex chromosomes at leptotene persists until first anaphase. In this species initial association depends on the characteristic "stickiness" of heterochromatin for which there is clearly no substitute.

There are many other cases where completely differential and heteropycnotic sex chromosomes, not involved in nucleolar formation, are associated non-specifically at first prophase. But, so far as we know, in all these cases the association always lapses prior to the segregation 
of the $\mathrm{X}$ and $\mathrm{Y}$ components. We have personal experience of two of them, Dicranocephalus agilis (Hemiptera: Heteroptera) and Tipula maxima (Diptera: Tipulide), in both of which the first division is equational for the sex chromosomes. In the former (Lewis and Scudder, I958) the sex chromosomes become negatively heteropycnotic at first metaphase. This change is not accompanied by the organisation of a visible nucleolus though material may be lost from the chromosomes. The $\mathrm{X}$ and $\mathrm{Y}$ then fall apart and behave as univalents dividing equationally at first anaphase. Prophase association leads to the $\mathrm{X}$ and $\mathrm{Y}$ auto-orientating side-by-side at first metaphase and this proximity doubtless facilitates the formation of a "pseudo-bivalent" at the second division of meiosis. The general sequence of events in Tipula maxima is similar, though considerably more complicated (John, I957).

We would conclude therefore that the situation in our beetles is comparable to the above types in that initial association depends on the "stickiness" of heterochromatin, but that the organisation of a persistent nucleolus maintains this association and determines the reductional separation of the $\mathrm{X}$ and $\mathrm{Y}$ at first division. It is possible of course, that the real difference is not the presence or absence of a visible nucleolus but whether or not the materials which, in beetles, are organised into a nucleolus remain on the chromosomes or diffuse away. This brings us back to Forficula. If heterochromatic sex chromosomes not involved in nucleolar formation invariably fall apart the continued association of the sex chromosomes in Forficula may be associated with their nucleolar-type activity.

In the mouse the sex vesicle is the only nucleolar-like body present in the spermatocyte and it disappears at the end of diplotene (Ohno et al., 1957, 1959b). Of Microtus Sachs says " the vesicle surrounding the sex chromosomes still persists at diplotene and diakinesis, but it has in most cases disappeared when the autosomes reach first metaphase". In the rat (Ohno et al., 1956, I958, I959a) the spermatocytes also include autosomal nucleoli and although these disappear " before or during pachytene" the sex vesicle persists through diplotene: clearly it is different in some way. This appears to be the case in Forficula too (cf. figs. I-3 of text-fig. 2, Callan, I94I). If negative heteropycnosity can indicate a loss of material it is significant that the sex chromosomes of Forficula, unlike those of Dicranocephalus and Tipula, do not show this property. It is possible therefore that they retain some part of the nucleolar material and that this accounts for their metaphase association.

The factors responsible for the association of the sex chromosomes in Drosophila have long been disputed. Here the paired regions include the nucleolar organisers and it has been suggested (Ohno et al., 1957) that a remnant of the nucleolar material plays a role in maintaining the association of $\mathrm{X}$ and $\mathrm{Y}$ chromosomes at least until the end of diakinesis. Perhaps the diffusion of such materials to the chromosome ends can account for " terminal affinity". 
Finally persistent nucleoli of altered chemical composition have been described in many grasses. These do not stain in the postprophase stages when aceto-carmine is used after the usual acid fixatives. They appear to be largely histone-containing, having lost their RNA (Brown and Emery, 1957). It may be therefore that the persistent nucleoli in beetles are distinctive only in the retention of this component beyond prophase.

\section{(ii) The situation in other Coleopterans}

A nucleolus associated with the sex chromosomes at prophase of meiosis has been described in the spermatocytes of a number of beetles, e.g., the curculionids Sitophilus granarius, Sitophilus oryze and Sitonia lepidus (Smith, 1952a) and the tenebrionids Tribolium castaneum, Tenebrio molitor, Scotobates calceratus and Tribolium confusum (Smith, $\left.\mathrm{r} 95^{2 b}\right)$; all but the last two of these have an $\mathrm{X} y_{p}$ system. In these cases the nucleolus has not been described as present at metaphase but Smith invariably describes and figures the $\mathrm{X}_{p}$ bivalent as being negatively heteropycnotic at this stage. We have never found this condition. It is true that the nucleolus stains less deeply and a difference in interpretation may explain the disparity since small $\mathrm{X} y_{p}$ bivalents are doubtless very difficult to analyse. We have no reason to doubt therefore that the nucleolus in other $\mathrm{X} y_{p}$ coleopterans plays a role comparable with that in the species we have studied.

Smith (1956) has described one case where the nucleolus does persist until metaphase. This was in Agriotes mancus, an elaterid beetle with an XX/XO sex-chromosome mechanism. Clearly, the nucleolus here cannot play the role that it does in ladybirds: it would have lost its function with the loss of the $y_{p}$ chromosome. Perhaps in this species we are dealing with a relatively recent evolution of the $\mathrm{XO}$ condition. The nucleolus probably lacks its mechanical function too in the comparatively recent neo-XX/XY system of Tribolium confusum but is indispensable in the phylogenetically older $\mathrm{X} n \mathrm{X} n / \mathrm{X} n \mathrm{Y}$ systems like those in the genus Blaps. Thus, one of the conditions necessary for the successful evolution of the multiple sex-chromosome mechanism of Blaps was presumably present in the ancestral type.

The sex-chromosome-associated nucleolus may not be a "true nucleolus" in the sense that both sex chromosomes carry specific organisers responsible for organising nucleoli in physiologically active somatic cells. But rather a nucleolus with a special mechanical function needed and organised only in spermatocytes. This appears to hold for the sex vesicle in Microtus which has never been observed at mitosis (Sachs, 1953) and for the sex vesicle in Rattus which is produced in addition to autosomal nucleoli (Ohno et al., r959a). This view would help to overcome the difficulty to which we have already drawn attention (Lewis and John, 1957), of explaining how all the sex chromosomes are involved in the organisation of the nucleolus in the spermatocytes of Blaps mucronata which has an $\mathrm{X}_{1} \mathrm{X}_{1} \mathrm{X}_{2} \mathrm{X}_{2} \mathrm{X}_{3} \mathrm{X}_{3} / \mathrm{X}_{1} \mathrm{X}_{2} \mathrm{X}_{3} \mathrm{Y}$ mechanism. 
Some evidence in support of this idea comes from the observation of secondary constrictions in the autosomes of Adalia bipunctata (text-fig. 3). Smith and Edgar (1954) on the other hand have described a satellited $y$ chromosome in Cicindela but the evidence is not compelling. It may be of course that some coleopterans are like Mus and others like Rattus or Forficula.

(iii) Some phylogenetic implications of the subsidiary devices

It was argued above that where the homologous segments of the sex chromosomes were short, ensuring their regular pairing may present

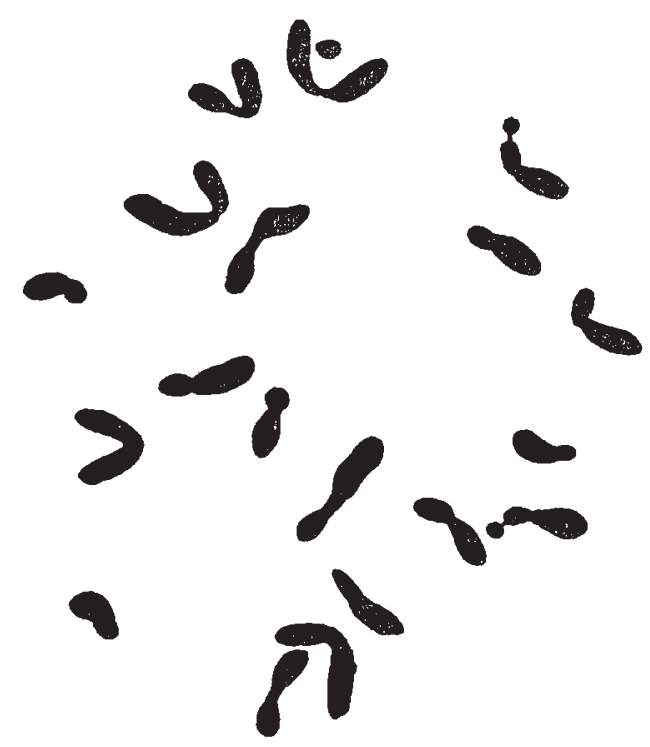

TExT-FIG. 3.-Coccinella septempunctata. Spermatogonial metaphase. Note presence of a pair of satellited autosomes. $\times 4500$ (Acetic orcein).

difficulty. It was further argued that other devices may facilitate initial contact in such cases and that one of these was the fusion of nucleolar-type organelles. While this may help to solve one problem it introduces another. Although it may help to bring chromosomes together it must surely impede the intimate pairing required for crossing over. Indeed Upcott (I936) has already shown that in both Fritillaria and Eremurus a nucleolus can reduce the frequency of chiasmata.

It may well be therefore that this device speeds up the very process that favoured its occurrence, namely the conversion of pairing into differential segments. As we have seen the differential segments of Microtus are extensive, and although the mouse has been the subject of intensive breeding experiments partial sex-linked determinants have yet to be found. Perhaps the nucleolus in these types will be ultimately called upon to serve the same function it now performs in ladybirds. 
One would think that the same argument could be applied in those cases where the sex chromosomes are positively heteropycnotic when they associate. This too is the case in mammals and in at least some of these crossing over in precociously condensed segments has been claimed to occur. It is true that Ohno et al. (1959b) do describe a stage during the prophase of meiosis in the mouse where the sex chromosomes in the sex vesicle become negatively heteropycnotic and maximally extended (cf. John and Lewis, I959). But it appears that the chromosomes are bipartite by now and that their terminal association has already been established by this time. Since the absence or infrequency of crossing over in precociously condensed segments has been recorded in so many other organisms the sex chromosomes of mammals are enigmatical in this respect and the situation requires clarification.

\section{SUMMARY}

I. In Adalia bipunctata, Coccinella septempunctata and Tenebrio molitor there is an $\mathrm{X} y_{p}$ sex-bivalent in the male. This bivalent develops precociously as a result of the association of the positively heteropycnotic $\mathrm{X}$ and $y_{p}$ chromosomes at pre-meiosis and is maintained until anaphaseI by the development during pachytene of a persistent nucleolus. This device enables the bivalent to achieve regular co-orientation in the absence of chiasmata though not infrequently the mechanical imposition of the persistent nucleolus leads to delayed congression. At first anaphase the $y_{p}$ detaches itself from the nucleolus and the sex chromosomes segregate.

2. We are of the opinion that this mechanism probably operates in all $\mathrm{XX} / \mathrm{X} y_{p}$ systems and, since it necessarily loses its function in simplified XX/XO systems, the retention of a persistent nucleolus in at least one of these (Agriotes mancus) is suggestive of its recent ancestry. It would appear therefore that one of the factors responsible for the proper functioning of multiple $\mathrm{X}_{n} \mathrm{X}_{n} / \mathrm{X}_{n} y$ mechanisms like those of Blaps was antecedent to such systems.

3. We suggest that nucleolar material can play a supplementary or even indispensable role in the association of largely or completely differential sex chromosomes either early (rodents) or late (Coleopterans) in the meiotic cycle. Moreover the material may (Coleopterans) or may not (Forficula, Drosophila?) be organised into a visible nucleolus.

Acknowledgment.-We are grateful to Miss Anne Templeton for collecting the material used in this study.

\section{REFERENCES}

BROWN, W. V., AND EMERY, W. H. P. 1957. Persistent nucleoli and grass systematics. Amer. F. Bot., 44, 585-59o.

CAllan, H. G. 1941. The sex-determining mechanism of the earwig, Forficula auricularia. F. genet., 4I, 349-374. 
All figs. $\times 4500$.

FIG. I.-Tenebrio molitor, early zygotene. Heteropycnotic $\mathrm{X} y_{p}$ association at ten o'clock. Note that six of the homologous autosomal centric $\mathrm{H}$-segments have begun to pair (aceto-carmine).

FIG. 2.- Tenebrio molitor, pachytene. $\mathrm{X} y_{n}$ association at twelve o'clock and now less condensed than the autosomal centric H-segments (aceto-carmine).

Figs. 3 and 4.-Tenebrio molitor, pachytene. Development of the $\mathrm{X} y_{p}$ associated nucleolus (aceto-carmine).

Fig. 5.-Coccinella septempunctata, metaphase-I. Typical parachute type sex bivalent (acetic orcein).

Fig. 6.-Adalia bipunctata, metaphase-I. Note the nucleolus between the $\mathrm{X}$ and $y_{p}$ chromosomes (aceto-carmine).

FIG. 7.-Tenebrio molitor, metaphase-I. Note $\mathrm{X} y_{p}$ with nucleolus but uncongressed (aceto-carmine)

FIG. 8.-Adalia bipunctata. Structure of the $\mathrm{X} y_{p}$ bivalent at metaphase-I (aceto-carmine).

FIG. 9.- Tenebrio molitor. Structure of the $\mathrm{X} y_{p}$ bivalent at metaphase-I (aceto-carmine).

FIG. Io.-Tenebrio molitor. Structure of the $\mathrm{X} y_{p}$ bivalent at early anaphase-I (acetocarmine).

FIG. 11.-Adalia bipunctata. Segregation of the $\mathrm{X}$ and $y_{p}$ chromosomes at anaphase-I. Note that the persistent nucleolus remains attached to the $\mathrm{X}$ (aceto-carmine). 
JoHn, в. 1957. XY segregation in the crane fly Tipula maxima (Diptera: Tipulide). Heredity, II, 209-2I5.

JOHN, B., AND LEWIS, K. R. 1959. Selection for interchange heterozygosity in an inbred culture of Blaberus discoidalis (Serville). Genetics, 44, 25I-267.

LEWIS, K. R., AND JoHN, B. I957. The organisation and evolution of the sex multiple in Blaps mucronata. Chromosoma, 9, 69-80.

LEWIS, K. R., AND SGUDDER, G. G. E. I958. The chromosomes of Dicranocephalus agilis (Hemiptera : Heteroptera). Cytologia, 23, 92-104.

ohno, s., KAPLAN, w. D., AND KINOSita, R. 1956. Concentration of RNA on the heteropycnotic XY bivalent of the rat. Exp. Cell. Res., II, 520-526.

OHNO, S., KAPLAN, W. D., AND KINOSITA, R. 1957. Note on non-chiasma type association between the $\mathrm{X}$ and $\mathrm{Y}$ chromosomes of Drosphila melanogaster and Mus musculus. Exp. Cell. Res., I3, 422-424.

OHNO, s., KAPLAN, W. D., AND KINOSITA, R. I958. A photographic representation of mitosis and meiosis in the male of Rattus norvegicus. Cytologia, 23, 422-428.

OHNo, S. KAPLAN, W. D., AND KINOSITA, R. I959a. The centromeriq and nucleolusassociated heterochromatin of Rattus norvegicus. Exp. Cell. Res., I6, 348-357.

OHNo, s., KAPLAN, W. D., AND KINDSiTA, R. I959b. On the end-to-end association of the $\mathrm{X}$ and $\mathrm{Y}$ chromosomes of Mus musculus. Exp. Cell. Res., I8, 282-29o.

SAcHs, L. I953. The giant sex chromosomes in the mammal Microtus agrestis. Heredity, 7, 227-238.

Sмiтh, s. G. I95I. Evolutionary changes in the sex chromosomes of Coleoptera. Genetica, 25, 522-524.

SMith, s. G. I $952 a$. The cytology of Sitophilus (Calandra) oryze (L.), S. Granarius. (L.) and some other Rhyncophora (Coleoptera). Cytologia, 17, 50-70.

Sмith, s. G. I952b. The cytology of some tenebrionid beetles (Coleoptera). F. Morph., $9 I, 325-364$.

smith, s. G. 1953. Chromosome numbers of Coleoptera. Herdity, 7, 31-48.

sмiтh, s. G. 1956. Spermatogenesis in an Elaterid beetle. 7. Hered., 47, 3-10.

SMITH, s. G., AND EDGAR, R. s. I954. The sex-determining mechanism in some North American Cicindelide (Coleoptera). Rev. Suisse de Zool., 6I, 657-667.

UPсотт, м. 1936. The origin and behaviour of chiasmata. XII. Eremurus spectabilis Cytologia, 7, I18-г 30.

\section{Note added in proof}

We have come across two additional relevant papers since our manuscript was submitted to press. First, N. M. stevens (1905), Publ. Carneigie Instit. Wash., 36, 3-7I described, under the term plasmosome, what is undoubtedly a neucleolar association between the $\mathrm{X}$ and $y p$ chromosomes of the beetles Odontata dorsalis, Trirhabda virgata and T. canadense. Secondly, L. sAchs (1954), Ann. Eugenics, I8, 255-26I, claims that in man the $\mathrm{X}$ and $\mathrm{Y}$ chromosomes are held together within a sex vesicle which forms before the autosomes reach pachytene. Generally one such vesicle is formed but the $\mathrm{X}$ and $\mathrm{Y}$ may organise individual vesicles which subsequently fuse. Sachs also suggests that it is a remnant of the sex vesicle that holds the sex chromosomes together at Metaphase-I and that no chiasma formation occurs. 